\title{
Stomatopsychology: A Newer Subject Bridging the Gap between Oral Health and Overall Health
}

\author{
Deepak Gupta* \\ Department of Oral Medicine and Radiology, M.M. College of Dental Sciences and Research, Mullana, Ambala, Haryana, India
}

Received: November 17, 2014; Accepted: November 19, 2014; Published: December 01, 2014

*Corresponding author: Dr. Deepak Gupta, Department of Oral Medicine and Radiology, M.M. College of Dental Sciences and Research, Mullana, Ambala, Haryana, India, Tel: +91-9896671281; E-mail: drdeepak_26@rediffmail.com

Oral Health is a window to the overall health of an individual. It has a great impact on the generalized systemic health. The state of the oral health can offer lot of clues about the overall health [1]. This calls for a need to learn more about this intimate connection between oral health and overall health. Overall health means complete physical, mental and social well being. Mental well being obviously is associated with brain or psychological state of an individual [1].

Interestingly the symptoms of anxiety and depression are getting commoner in the community [1-3]. Gupta et al. [1] and Friedlander et al. [2] stated that dentists should be concerned with the assessment and identification of dental patients with psychological problems as it is extensively associated with dental disease. It is interesting to note that psychological illness was elicited by $57 \%$ of the patients with facial pain syndromes [3]. Moreover dental fear may also represent an underlying phobia or trait anxiety. Knowing this trait will definitely aid in better day to day treatment of dental patients.

\section{Etiology and Pathogenesis}

Psychological states influence all the body processes by three different mechanisms including neural, hormonal and immunologic [1,4]. Psyche or stress may lead to alteration of neurotransmitters \& hormones inside the individual's body. This alteration further results in translation of an emotion to either psychosomatic or somatoform disorder [1,4]. Psychosomatic disorder involves real physical illness and is capable of initiating or aggravating painful lesions inside the oral cavity. These lesions may include lichen planus, pemphigus, aphthous stomatitis, bullous pemphigoid, ANUG, lip licking cheilitis, Stevens Johnson's syndrome and cicatricial pemphigoid $[1,5]$. Otherwise patients may present with Somatoform disorders which may involve apparent physical illness without any organic basis to them. These constitute orofacial pain, burning mouth syndrome and temporomandibular disorders $[1,5]$. It is a well known fact that orofacial pain is a frequent cause of presentation to medical and dental practitioners. Although in majority of cases the cause is dental disease which can be easily diagnosed, the remaining are often difficult to diagnose and treat. Many researchers have found out a psychological parameter involved in these disorders and henceforth psychological treatment is included in the multimodal treatment approach of such disorders.

\section{Role of Dental Professionals}

As already discussed, the influence of psychological factors on an individual can be assessed as multi-factorial psychosomatic and/or somatoform disorders. Attention must also be paid to the fact that psychiatric illness may also develop as a result of skin/oral mucosal disease. Dental professionals lag behind understanding such cases as we do not regard ourselves as having a role in identifying dental patients with psychological problems. So there is a need to recognize emotional disorders in the physically ill persons [1]. Furthermore the dental professionals must be well versed with the knowledge regarding dental management of previously diagnosed cases of psychological illness. They must also be an expert regarding the knowledge of oral manifestations or complications of antipsychotic treatment. For example, oral dyskinesia which involves abnormal involuntary, uncontrollable movements affecting primarily the tongue, lips and jaws (can extend to trunk and limbs). It occurs due to conventional antipsychotics [6,7]. It may also lead to Neuropsychiatric conditions, oral ulcers, edentulousness, xerostomia, dysphagia, dysgeusia etc [6]. It has also been reported by several authors that $25 \%$ of patients on conventional antipsychotics have tardive dyskinesias after 5 years of treatment.

Simple screening questions regarding psychological assessment must be accommodated in the routine case history Performa if any of the psychosomatic or somatoform disorder is suspected so that they can be easily accessed by their response for these questions. Further observing the patient's mood and behavior during the dental appointment also provides a clue to psychological attitude. Screening questions about the previous psychiatric history and social factors must also be stressed. If emotional disorder is suspected then consideration must be given for full psychiatric history of the patient.

\section{Evidence of Mind Body Interaction}

An interesting piece of evidence for mind body connection is placebo as the patient makes genuine recovery from a sugar pill. 
This aspect is proved by many researchers in their randomized control trials. This explains the efficacy of faith healings and alternative medicines. More research is needed to explain the underlying mechanisms regarding this. Current research on psychosomatic disorders reveals that there are changes of 5-HT, Adreno Cortico Tropic Harmone (ACTH), IL-1 and IL-6 in diabetes mellitus patients, and they are correlated to anxiety, depression and glucose level [1,4]. After anxiolytic and antidepressant treatment, the changes in the above return to normal, and so is blood glucose level. Secondly, there is characteristic change of personality in diabetes mellitus patients that are somewhat same as that of depressive patients. Academic stress can lead to immunological changes and illness. Glaser et al. in 1985 assessed forty second year medical students 6 weeks before and during final exams. Lymphocytes, Natural Killer (NK) cells, NK cytotoxic activity and interferon levels were found lower [7].

\section{Role of Patient education}

No one chooses to have a mental disorder. Admitting to mental illness is not the same thing as admitting to any other serious health issue. This type of psychological attitude is more prevalent in developing countries as people consider it as a social stigma being considered as a mentally ill [1]. Further many studies have concluded that even after known stress/depressive factors involved, patients are quite reluctant to be referred for psychological assessment. Referral to a psychologist or a psychiatrist often results in more suspicion than support. Hence patients must be educated regarding the role of psychological factors involved in such diseases so as to check the misconceptions regarding these diseases [8].

\section{Conclusion}

Considering the involvement of psychology as a rootassociated factor, it can be concluded that there must be psychology training for the dental students. The dental professionals must be made aware of all these facts by continuing dental education programmes and other articles on the topic. Further there must be introduction of psychology as a separate subject with emphasis on oral psychosomatic disorders. This could separately be termed as "Somatopsychology" [1].

\section{References}

1. Gupta D, Sheikh S, Rashmi NC, Aggarwal A, Bansal R. Assessment of the Awareness of Dental Professionals Regarding Identification and Management of Dental Patients with Psychological Problems in Routine Dental Operatory: a Survey. Oral Health Dent Manag. 2014; 13(2): 435-40.

2. Friedlander AH, West LJ. Dental management of the patient with major depression. Oral Surg Oral Med Oral Patholo. 1991; 71(5): 573-8.

3. Lloyd-Williams F, Dowrick C, Hillon D, Humphris G, Moulding G, Ireland R. A preliminary communication on whether general dental practitioners have a role in identifying dental patients with mental health problems. Br Dent J. 2001; 191(11): 625-9.

4. Koh KB, Lee BK. Reduced lymphocyte proliferation and interleukin-2 production in anxiety disorders. Psychosom Med. 1998; 60(4): 47983.

5. Brightman VJ. Oral symptoms without apparent physical abnormalityAtypical facial pain, glossodynia and burning mouth syndrome, subjective xerostomia, and idiopathic dysguesia. In: Lynch MA, Brightman VJ, Greenberg MS, Editors. Burket's Oral Medicine Diagnosis and Treatment. 9th ed. Philadelphia: Lippincott- Raven; 1994. p. 379-414.

6. LM Abetz, NW Savage. Burning mouth syndrome and psychological disorders. Aust Dent J. 2009; 54(2): 84-93. doi: 10.1111/j.18347819.2009.01099.x

7. Bertini F, CostaNC, Brandão AA, Cavalcante AS, Almeida JD. Ulceration of the oral mucosa induced by antidepressant medication: a case report. J Med Case Rep. 2009, 3: 98. doi: 10.1186/1752-1947-3-98.

8. Gupta D, Sheikh S, Pallagatti S, Kasariya K, Buttan A, Gupta M. Burning mouth syndrome due to television moans, an enigma for oral physician: Treatment with counselling. J Dent Res Dent Clin Dent Prospect. 2014; 8(2): 118-122 